\title{
Argonaute-2 regulates the proliferation of adult stem cells in planarian
}

\author{
Yong-Qin $\mathrm{Li}^{1,3}$, An Zeng ${ }^{1,3}$, Xiao-Shuai Han ${ }^{1}$, Chen Wang ${ }^{1}, \mathrm{Ge} \mathrm{Li}^{1,4}$, Zhen-Chao Zhang ${ }^{1}$, Jian-Yong Wang ${ }^{1}$, \\ Yong-Wen Qin ${ }^{2}$, Qing Jing ${ }^{1,2}$ \\ ${ }^{I}$ Key Laboratory of Stem Cell Biology and Laboratory of Nucleic Acid and Molecular Medicine, Institute of Health Sciences, \\ Shanghai Institutes for Biological Sciences, Chinese Academy of Sciences and Shanghai Jiao-Tong University School of Medicine, \\ Shanghai 200025, China; ${ }^{2}$ Department of Cardiology, Changhai Hospital, Shanghai 200433, China; ${ }^{3}$ Graduate School of Chinese \\ Academy of Sciences, Beijing 100049, China; ${ }^{4}$ Present address: Department of Pediatrics, University of Alberta, Edmonton, AB, \\ Canada T6G $2 S 2$
}

Cell Research (2012) 22:275. doi:10.1038/cr.2012.8; published online 5 January 2012

Correction to: Cell Research (2011) 21:1750-1754. doi:10.1038/cr.2011.151; published online 6 September 2011

In our discussion of the role of Argonaute proteins in stem cell regulation, we inadvertently omitted an important earlier contribution by Agata's group [1]. Through testing conserved post-transcriptional regulators in the planarian Dugesia japonica, their study reports that Djago 2 is required to maintain stem cell identity.

We regret any inconvenience caused by this omission.

\section{Reference}

1 Rouhana L, Shibata N, Nishimura O, Agata K. Different requirements for conserved post-transcriptional regulators in planarian regeneration and stem cell maintenance. Dev Biol 2010; 341:429-443. 